\title{
Satisfacción laboral: una breve revisión
}

Margarita Peya Gascóns

Profesora Titular de Administración de los Servicios de Enfermería. Escuela de Enfermería. Universidad de Barcelona. Barcelona. España.

En la presente era en la que imperan las presiones para

la contención de los costes, se hace necesario que los profesionales enfermeros gestores garanticen que los profesionales enfermeros asistenciales tengan un entorno de trabajo estrechamente relacionado con la satisfacción laboral, de forma que se logren buenos resultados, fruto de una óptima mejora de la calidad de los cuidados enfermeros prestados.

\section{CONCEPTO DE SATISFACCIÓN LABORAL}

La satisfacción laboral es un tema que cuenta con un amplio interés y es una de las variables de estudio más frecuentes en investigaciones relativas a los fenómenos relacionados con las teorías organizativas y los diseños de puestos de trabajo ${ }^{1}$. El modelo tradicional de satisfacción laboral se centra en las percepciones que una persona tiene respecto a su trabajo. Sin embargo, lo que produce satisfacción o insatisfacción laboral no depende de la naturaleza del trabajo, pero sí de las expectativas que se cree que el trabajo puede proporcionar ${ }^{2}$.

El trabajo de Maslow (1954) sugiere que las necesidades humanas conforman una jerarquía de 5 niveles que van desde las necesidades fisiológicas de seguridad, pertenencia y autoestima a las de autorrealización. Basándose en la teoría de Maslow, la satisfacción en el trabajo ha sido enfocada por los investigadores desde la perspectiva de una necesidad de realización ${ }^{3}$. Sin embargo, este enfoque ha llegado a ser menos popular con el incremento del énfasis puesto en el proceso cognitivo más que en subrayar las necesidades que desde una perspectiva actitudinal han llegado a ser predominantes en los estudios de satisfacción laboral.

En contraste con la visión tradicional, Herbertz y Mausner (1959) formularon la teoría de 2 factores relacionados con la satisfacción laboral y postularon que la satisfacción y la insatisfacción estaban separadas y algunas veces eran fenómenos no relacionados. Los factores

Este artículo forma parte del proyecto de investigación "Satisfacción laboral de los profesionales enfermeros, en relación con el modelo de gestión hospitalario", que en su día constituirá una tesis doctoral.

intrínsecos, llamados motivadores, estaban basados en los satisfactores del trabajo e incluían el logro, el reconocimiento, el trabajo en sí mismo y la responsabilidad. Los factores extrínsecos, llamados factores higiénicos, estaban basados en los factores que producen insatisfacción en el trabajo e incluían las políticas institucionales, la administración, la supervisión, el salario, las relaciones interpersonales y las condiciones de trabajo. La teoría de higiene y motivación de Herbertz y Mausner ha prevalecido en los estudios de satisfacción de la naturaleza del trabajo y ha conformado las bases del desarrollo de la valoración de dicha satisfacción laboral. De este modo, la satisfacción en el trabajo es una orientación afectiva que un empleado tiene hacia su trabajo ${ }^{4}$. Puede ser considerada como un sentimiento global acerca del trabajo o como una constelación de actitudes relacionadas con varios aspectos o facetas del trabajo ${ }^{1}$. Spector resumió las siguientes facetas de la satisfacción laboral: reconocimiento, comunicación, colaboradores, beneficios, sueldo, desarrollo personal, oportunidades de promoción, seguridad y supervisión.

En la actualidad también se considera que la satisfacción de los profesionales enfermeros está relacionada con las condiciones de trabajo, las relaciones que comporta el puesto, el propio puesto de trabajo, el reconocimiento, la 
remuneración, el autodesarrolo y la promoción, el nivel de responsabilidad y seguridad, así como el estilo de liderazgo y las políticas y protocolos organizativos $^{5}$.

\section{FUENTES DE SATISFACCIÓN LABORAL DE LOS PROFESIONALES ENFERMEROS}

Teniendo en cuenta que la satisfacción laboral está relacionada con una práctica de óptima calidad ${ }^{6}$, no sorprende que el concepto de satisfacción en el trabajo haya sido objeto de diferentes estudios.

Los investigadores han intentado identificar los diferentes componentes de la satisfacción laboral y examinar qué efecto tenían dichos componentes en la productividad de los empleados ${ }^{7}$. En la literatura enfermera se pueden encontrar una serie de hallazgos derivados de estudios tanto cuantitativos como cualitativos sobre este tema.

Un estudio multicéntrico que abarca diferentes países ${ }^{8}$ muestra que la insatisfacción más elevada entre los profesionales enfermeros se daba en Estados Unidos (41\%), seguido de Escocia (38\%), Inglaterra (36\%), Canadá (33\%) y Alemania (17\%). En el momento de la recogida de datos, un tercio de las enfermeras de Escocia e Inglaterra y más de una quinta parte de las de Estados Unidos planeaban dejar su trabajo. En relación con el clima laboral, sólo un tercio de las enfermeras de Canadá y
Escocia tenían la percepción de que participaban en el desarrollo organizativo de su trabajo. El estudio también muestra que el $61 \%$ de los profesionales enfermeros de Alemania están menos satisfechos con las oportunidades de desarrollo que los de Estados Unidos (57\%) y Canadá (69\%), que se sentían más satisfechos con su salario.

Otro estudio similar ${ }^{9}$ halló que los profesionales enfermeros británicos se perciben menos satisfechos que sus homónimos australianos ( $<<0,001)$. profesionales de la salud, entre ellos los médicos y los gestores $(p<0,01)$.

Uno de los aspectos más relevantes patente en diferentes investigaciones relacionadas con la satisfacción laboral de los profesionales enfermeros es la estructura organizativa ${ }^{10}$. Otros autores ${ }^{11}$ consideran que la estructura organizativa es un determinante crucial en la satisfacción laboral de los profesionales enfermeros.

Diferentes estudios relacionados con la satisfacción laboral de los profesionales

La satisfacción laboral de los profesionales enfermeros es un concepto importante que puede incidir en los aspectos relacionados con la retención -y por tanto en la estabilidad de las plantillas en el hospital- y es a su vez un concepto clave en la mejora de la calidad de los cuidados que se proporcionan a los pacientes.

Los profesionales enfermeros británicos perciben su estatus profesional bajo $(\mathrm{p}<0,01)$, sus relaciones con los gestores de los hospitales pobres $(\mathrm{p}<0,01)$ y sus condiciones de trabajo menos adecuadas que los australianos $(\mathrm{p}<0,01)$. También informan que existe un mayor conflicto entre la perspectiva idealizada del trabajo adquirida en los estudios y la práctica actual ( $p<0,01)$, y que están menos satisfechos con su organización profesional $(p<0,01)$. Los profesionales enfermeros británicos están también muy preocupados por la falta de comunicación con los médicos $(p<0,01)$ e informan sentir menos respeto por parte de otros enfermeros muestran variadas fuentes de satisfacción. Los hallazgos derivados de diferentes investigaciones que utilizan instrumentos de medición similares son más valiosos, ya que proporcionan información comparada. Por ejemplo, Adams et al $^{12}$ utilizan la Ward Organisational Features Scales en una serie de estudios en los que recogen información de las percepciones de los profesionales enfermeros con respecto a diferentes aspectos de su trabajo en la unidad/servicio y el impacto que tiene en la organización de los cuidados. El instrumento consta de 6 grupos de mediciones que comprenden 14 
subescalas con una escala de Likert de 4 puntos (1: totalmente en desacuerdo/muy $\mathrm{mal} /$ muy dificultoso; 4: totalmente de acuerdo/muy bien/muy fácil): el entorno físico de la unidad/servicio, la práctica enfermera profesional, las relaciones en el trabajo, el liderazgo en la unidad, la influencia de los profesionales enfermeros y la satisfacción laboral.

Utilizando este cuestionario, Tovey y Adams ${ }^{13}$ hallaron que las fuentes principales de insatisfacción de los profesionales enfermeros incluían las relaciones laborales, especialmente las relacionadas con la gestión, la falta de plantilla, la preocupación profesional respecto a bajos estándares de cuidados y la presión externa del trabajo. Un estudio ${ }^{14}$ muestra también que los profesionales enfermeros indicaron como aspectos positivos de la unidad/servicio las facilidades y el diseño. Las correlaciones componentes laborales: autonomía, estatus profesional, sueldo, interacción, protocolos organizativos y

requerimientos de las tareas que utiliza Index of Work Satisfaction ${ }^{19}$. La primera parte se dirige a la relativa importancia de cada uno de los 6 componentes del trabajo, utilizando 15 grupos de criterios. La segunda parte es una escala de Likert de 44 ítems, que requiere efectuar de 1 a 7 elecciones de totalmente en desacuerdo a totalmente de acuerdo para medir niveles corrientes de satisfacción relacionados con cada uno de los 6 componentes. Los resultados muestran que los profesionales enfermeros están más insatisfechos que satisfechos ( significado $=3,46)$ y muestran mayor satisfacción con el estatus profesional ( significado $=4,17$ ) y mayor grado de insatisfacción con los requerimientos para las tareas (significado $=2,81$ ).

Muchos estudios de investigación revelan que la satisfacción de los profesionales enfermeros en el hospital está estrechamente relacionada con el nivel de autonomía, las condiciones laborales, el respeto y reconocimiento al trabajo que se realiza, una plantilla adecuada y suficiente, las buenas relaciones entre los miembros del equipo, el sueldo, el compromiso con la organización y la implicación profesional.

más elevadas se hallaron entre la satisfacción del trabajo y la cohesión del equipo de enfermería $(r=0,51 ; p<0,00)$, la organización de la plantilla $(r=0,46 ; p$ $<0,001$ ), el nivel de práctica profesional lograda en la unidad ( $r=0,46 ; p<0,001)$ y la colaboración con el equipo médico $(\mathrm{r}=0,41 ; \mathrm{p}<0,01)$.

Otros autores ${ }^{15-17}$ utilizan un cuestionario de satisfacción en una serie de estudios llevados a término en diferentes países para valorar la satisfacción y el nivel de motivación de los profesionales enfermeros. Dicho estudio comprende 16 ítems que se dirigen a aspectos del entorno laboral, la percepción de los cambios en los últimos 12 meses y la completa satisfacción y motivación. Las respuestas se contabilizan en una escala Likert de 5 puntos (totalmente de acuerdo/incrementa muchísimo a totalmente en desacuerdo/disminuye muchísimo).

Otro punto de vista lo aporta un trabajo $^{18}$ que examina el nivel de satisfacción laboral en relación a 6
Un estudio realizado en 3 hospitales del sur de Taiwán pone de manifiesto que las expectativas y la realidad pueden ser una fuente de insatisfacción entre los profesionales enfermeros. Se elaboró un cuestionario sobre la importancia de la percepción y la satisfacción de los profesionales enfermeros que fue especialmente diseñado para dicho estudio e incluía 92 ítems que comprendían 8 componentes sobre satisfacción laboral y 8 indicadores de importancia (entorno de trabajo directo, entorno de trabajo indirecto, sueldo y promoción, autodesarrollo, trabajo desafiante, interacción con los pacientes, estilo de liderazgo y clima laboral). Las repuestas se dieron en una escala de Likert de 5 puntos en un rango de 1 (menos satisfecho) a 5 (muy satisfecho). La correlación de Pearson analizada reveló que, excepto para el indicador de entorno de trabajo indirecto, todos los componentes se correlacionaban estadísticamente y significativamente con sus correspondientes componentes
( $p \leq 0,05)$. La muestra de profesionales enfermeros identificó factores tales como el entorno de trabajo indirecto (protocolos del hospital, beneficios, actividades de ocio, políticas vacacionales, entre otros), el salario y la promoción como importantes pero muy insatisfactorios ${ }^{20}$.

En conclusión, la satisfacción laboral de los profesionales enfermeros es un concepto importante que puede incidir en los aspectos relacionados con la retención -y por tanto en la estabilidad de las plantillas en el hospital- y es a su vez un concepto clave en la mejora de la calidad de los cuidados que se proporcionan a los pacientes.

\section{FACTORES RELACIONADOS CON LA SATISFACCIÓN LABORAL DE LOS PROFESIONALES ENFERMEROS}

La satisfacción del trabajo es un fenómeno complejo, como se ha evidenciado en el apartado anterior. La identificación de los factores relacionados y el examen de sus efectos pueden ayudar a desarrollar las intervenciones de gestión necesarias para mejorar la calidad de los cuidados.

Un modelo causal de satisfacción laboral que ha sido probado implicó a profesionales enfermeros de Taiwán y de Corea del Sur. Dicho modelo incluye 11 variables independientes: entorno laboral, afectividad positiva, afectividad negativa, autonomía, justicia distributiva, justicia procesal, estrés laboral, sueldo, cambios promocionales, rutinización y apoyo social. Los investigadores ${ }^{21}$ hallaron que, con excepción del sueldo, todas las variables estaban significativamente relacionadas con la satisfacción laboral.

El compromiso con la organización se refiere a la identificación con la lealtad a la institución y a sus objetivos ${ }^{22,23}$ y se ha definido como una fuerza de identificación de la persona y su implicación en la organización. En particular, el compromiso se caracteriza por 3 factores: una fuerte creencia y aceptación de los objetivos y valores de la organización, la voluntad para ejercer un esfuerzo considerable en nombre de la organización y un fuerte deseo de mantenerse miembro de la organización. 
Se ha hallado que el compromiso organizativo es positivo en relación con la satisfacción de los profesionales enfermeros que ejercen en el hospital 24,25 . Un estudio realizado a una muestra de profesionales enfermeros canadienses halló que el compromiso organizativo estaba relacionado con la satisfacción global del trabajo $(r=0,64$; $\mathrm{p}<0,001$ ), la satisfacción con el trabajo $(r=0,058 ; p<0,001)$, las oportunidades de promoción $(r=0,055 ; \mathrm{p}<0,001)$, la supervisión $(r=0,054 ; p<0,001)$, los colaboradores $(r=0,030 ; p<0,001)$ y el sueldo $(r=0,022 ; p<0,001)$. Además, el análisis de regresión revelaba que el compromiso organizativo explicaba el $41 \%$ de la varianza en la satisfacción laboral ${ }^{26}$

El compromiso profesional supone la implicación de la persona hacia su profesión. En enfermería, dicho compromiso tiene el potencial de producir beneficios tanto para el profesional como para la organización. Además, dicho compromiso profesional está positivamente relacionado con la satisfacción laboral de los profesionales enfermeros ${ }^{22,27}$. Por ejemplo, un estudio realizado en Singapur ${ }^{28}$ demostró que la satisfacción en el trabajo de los profesionales enfermeros estaba significativa y positivamente relacionada con el compromiso profesional.

Los roles de los profesionales enfermeros en su lugar de trabajo respecto a conflicto y ambigüedad pueden llegar a ser una fuente de estrés. El rol conflictivo es definido en términos de las dimensiones de congruenciaincongruencia, o compatibilidadincompatibilidad en los requerimientos del rol. El rol conflictivo y el ambiguo están relacionados con el estrés laboral, el compromiso organizativo, la satisfacción laboral y la intención de dejar la profesión enfermera ${ }^{29}$.

Sin embargo, otro estudio ${ }^{30}$ muestra que no hay una asociación lineal entre la satisfacción laboral de los profesionales enfermeros y la correlación entre los valores del rol laboral y los roles del trabajo actual $(p<0,05)$. Pero los roles utilizados en el cuestionario no fueron validados.

Un estudio muy interesante $e^{31}$ es el que analiza el impacto de la estructura organizativa en la satisfacción laboral de los profesionales enfermeros. Para ello se pasó un cuestionario a una muestra de 764 profesionales enfermeros de 3 hospitales belgas. Se utilizó la importancia de las 6 dimensiones de la satisfacción laboral de acuerdo a Stamps y Piedmonts y se vio que, de mayor a menor, el porcentaje de importancia fue el siguiente: salario $(69,2 \%)$, autonomía $(58,9 \%)$, interacción $(49,3 \%)$, status $(43,2 \%)$, requerimientos para las tareas $(42,4 \%)$, protocolos organizativos.

En resumen, se puede decir que muchos estudios de investigación revelan que la satisfacción de los profesionales enfermeros en el hospital está estrechamente relacionada con el nivel de autonomía, las condiciones laborales, el respeto y reconocimiento al trabajo que se realiza, una plantilla adecuada y suficiente, las buenas relaciones entre los miembros del equipo, el sueldo, el compromiso con la organización y la implicación profesional. Aspectos todos estrechamente relacionados con el estilo y modelo de gestión establecido por los directivos de la organización.

\section{Bibliografía}

1. Spector PE. Job satisfaction application, assessment, causes, and consequences. London: SAGE; 1997.

2. Lu H, While A, Barriball L. Job satisfaction and its related factors: A questionnaire survey of hospital nurses in Mainland China. Int J Nurs Stud. 2007; 44:574-88.

3. Conrad KM, Conrad KJ, Parker JE. Job satisfaction among occupational health nurses. J Community Health Nurs. 1985;161-73.

4. Price JL, Mueller CW. Professional turnover. The case for nurses. Iowa State: University Press Ames; 1981.

5. Cavanagh SJ. Job-satisfaction of nursing staff working in hospitals. J Adv Nurs. 1992;17:704-11.

6. Landeweerd JA, Boumans NPG. Nurses' work satisfaction and feelings of health and stress in three psychiatric departments. Int J Nurs Stud. 1988;25:22534.

7. Burnard P, Morrison P, Phillips C. Job satisfaction amongst nurses in an interim secure forensic unit in Wales. Aust N Z J Ment Health Nurs. 1999;8:9-18.

8. Aiken L, Clarke S, Sloane D, Sochalski J, Busse R, Clarke H, et al. Nurses' reports on hospital care in five countries. Health Aff. 2001;20:43-53.

9. Adamson B, Kenny D, Wilson-Barnett J. The impact of perceived medical dominance on the workplace satisfaction of Australian and British nurses. J Adv Nurs. 1995;21:172-83.

10. Campbell L, Fowles ER, Weber BJ. Organizational structure and job satisfaction in public health nursing. Public Health Nurs. 2004;6:564-71.

11. Cumbey DA, Alexander JW. The relationship of job satisfaction with organizational variables in public health nursing. J Nurs Adm. 1998;28:39-46.

12. Adams A, Bond S, Arber S. Development and validation of scales to measure organisational features of acute hospital wards. Int J Nurs Stud. 1995;32:612
13. Tovey E, Adams A. The changing nature of nurses job satisfaction an exploration of sources of satisfaction in the 1990s. J Adv Nurs. 1999;30:150-8.

14. Adams A, Bond S. Hospital nurses' job satisfaction, individual and organizational characteristics. J Adv Nurs. 2000;32:536-43.

15. Nolan M, Nolan J, Grant G. Maintaining nurses' job satisfaction and morale. Br J Nurs. 1995;19:114854.

16. Nolan M, Brown J, Naughton J, Nolan J. Developing nursing's future role 2 nurses' job satisfaction and morale. Br J Nurs. 1998;17:1044-8.

17. Lundh U. Job satisfaction among Swedish nurses and laboratory technologists. Br J Nurs. 1999;14:94852.

18. Lee FK. Job satisfaction and autonomy of Hong Kong registered nurses. J Adv Nurs. 1998;27:355-63. 19. Stamps PL, Piedmonte PB. Nurses and work satisfaction. An index for measurement. USA: Health Administrative Press; 1986

20. Tzeng HM. The influence of nurses' working motivation and job satisfaction on intention to quit an empirical investigation in Taiwan. Int J Nurs Stud. 2002;9:867-78

21. Chu CI, Hsu HM, Price JL, Lee JY. Job satisfaction of hospital nurses an empirical test of a causal model in Taiwan. Int Nurs Rev. 2003;50:176-82.

22. Blau GJ, Lunz M. Testing the incremental effect of professional commitment on intent to leave one's professional beyond the effects of external, personal and work-related variables. J Vocat Behav. 1998; $52: 260-9$.

23. Mowday RT, Steers RM, Porter LM. The measurement of organizational commitment. J Vocat Behav. 1979;14:224-7

24. Blegen M. Nurses' job satisfaction a meta-analysis of related variables. Nurs Res. 1993;42:36-41.

25. Al-Aameri AS. Job satisfaction and organizational commitment for nurses. Saudi Med J. 2000;21:531-5. 26. Knoop R. Relationships among job involvement, job satisfaction and organizational commitment for nurses. J Psychol. 1995;129:643-7.

27. Cohen A. An examination of the relationship between work commitment and work outcomes among hospital nurses. Scand J Manage. 1998;14:1-17.

28. Rosse JG, Rosse PH. Role conflict and ambiguity an empirical investigation of nursing personnel. Eval Health Prof. 1981;4:385-405

29. Fang Y. Turnover propensity and its causes among Singapore nurses an empirical study. Int J Hum Resour Manage. 2001;12:859-71.

30. Mitchell MB. The effect of work role values on job satisfaction. J Adv Nurs. 1994;20:958-63.

31. Willem A, Buelens M, De Jonghe I. Impact of organizational structure on nurses' job satisfaction: A questionnaire survey. Int J Nurs Stud. En prensa, 2007. Disponible en:

http://www.feb.ugent.be/fac/research/WP/Papers/wp_0 5_347.pdf

Correspondencia: M. Peya Gascons.

Escola d'Infermeria de la Universitat de

Barcelona.

Pabelló de govern. Feixa Llarga, s/n. 08907

L'Hospitalet de Llobregat. Barcelona. España

Correo electrónico: mpeya@ub.edu 